\title{
Experimental teaching and training system based on volume holographic storage
}

\author{
Zhuqing Jiang, Zhe Wang, Chan Sun, Yutong Cui, Yuhong \\ Wan, et al.
}

Zhuqing Jiang, Zhe Wang, Chan Sun, Yutong Cui, Yuhong Wan, Rufei Zou, "Experimental teaching and training system based on volume holographic storage," Proc. SPIE 10452, 14th Conference on Education and Training in Optics and Photonics: ETOP 2017, 104521V (16 August 2017); doi: 10.1117/12.2270721

SPIE Event: 14th Conference on Education and Training in Optics and Photonics, ETOP 2017, 2017, Hangzhou, China 


\title{
Experimental teaching and training system based on volume holographic storage
}

\author{
Zhuqing Jiang ${ }^{1,2}$, Zhe Wang ${ }^{1}$, Chan Sun $^{1}$, Yutong Cui ${ }^{1}$,Yuhong Wan ${ }^{1,2}$, Rufei Zou ${ }^{1}$ \\ 1. College of Applied Sciences, Beijing University of Technology, Beijing 100124, China \\ 2. Institute of Information Photonics Technology, Beijing University of Technology, Beijing 100124, China
}

\begin{abstract}
The experiment of volume holographic storage for teaching and training the practical ability of senior students in Applied Physics is introduced. The students can learn to use advanced optoelectronic devices and the automatic control means via this experiment, and further understand the theoretical knowledge of optical information processing and photonics disciplines that have been studied in some courses. In the experiment, multiplexing holographic recording and readout is based on Bragg selectivity of volume holographic grating, in which Bragg diffraction angle is dependent on grating-recording angel. By using different interference angle between reference and object beams, the holograms can be recorded into photorefractive crystal, and then the object images can be read out from these holograms via angular addressing by using the original reference beam. In this system, the experimental data acquisition and the control of the optoelectronic devices, such as the shutter on-off, image loaded in SLM and image acquisition of a CCD sensor, are automatically realized by using LabVIEW programming.
\end{abstract}

Keywords: Volume holographic storage, angle-multiplexing, Bragg selectivity, photorefractive crystals, holographic reconstruction

\section{INTRODUCTION}

Optical volume holographic storage is promising for the next-generation storage, optical interconnection, optical neural network, and other relevant fields because of the potential high-density, large-capacity, fast data transfer rate, and the possibility of fully parallel readout. Photorefractive crystals are widely used as storage media owing to their advantages such as relatively large thickness, no shrinkage, recyclability and so on. The phase type of holograms formed in photorefractive crystals of several millimeters thickness can give high diffraction efficiencies and high storage capacities. Storage of thousands of holograms had been demonstrated in $1990 \mathrm{~s}^{[1,2]}$, and the density up to $387 \mathrm{bit} / \mathrm{mm}^{2}$ was demonstrated in 2001 by storing 1000 high-resolution data pages in a small location of a storage medium ${ }^{[3]}$. The relevant theory and realizing means of photorefractive holographic storage are completed, and its experiment setup consists of various optoelectronic devices that are developed in recent decades. Thus, the experiment of volume holographic recording and readout is a comprehensive experimental project that is set up for senior undergraduate students majoring in applied physics or in optical engineering. This experiment project is rebuild and simplified from a research-level experiment, in which a variety of knowledge in optics and photonics, such as optical interference and diffraction, optical information processing, crystal optics and some concepts in laser, will be involved. It is effective for training and education of practical ability in optical technology. The experiment of volume holographic storage consists of the

14th Conference on Education and Training in Optics and Photonics: ETOP 2017, edited by Xu Liu,

Xi-Cheng Zhang, Proc. of SPIE Vol. 10452, 104521V • (c) 2017 ICO, IEEE, OSA, SPIE

CCC code: $0277-786 X / 17 / \$ 18 \cdot$ doi: $10.1117 / 12.2270721$

Proc. of SPIE Vol. 10452 104521V-1 
knowledge of optical information processing and photonics disciplines, relevant experiment skills, and the basic method of data acquisition and processing. The student can learn to use advanced optoelectronic devices and the basic automatic control means.

In this paper, the principle and experimental configuration of multiplexing holographic recording and readout based on Bragg selectivity of volume holographic grating is introduced. In this experiment, the data acquisition and the control of the optoelectronic devices, such as the shutter on-off, image loaded in SLM and image acquisition of a CCD sensor, are automatically realized via a user interface with LabVIEW programming.

\section{PRINCIPLE OF VOLUME HOLOGRAPHIC STORAGE}

Volume holograms can be used as optical memories, interference filters, multiplexers, pattern recognition, and other diffraction devices. Photorefractive crystals such as $\mathrm{Fe}: \mathrm{LiNbO}_{3}$ or Fe:Ce: $\mathrm{LiNbO}_{3}$ are commonly employed as a kind of recording medium for volume holography, into which the volume holographic gratings (holograms) are written via the electronic charge excitation and transport mechanism ${ }^{[4,5]}$. A reference beam and an object beam interfere within a photorefractive crystal to form interference fringe, termed as a volume hologram. If the reference beams with different incidence angles interfere with an object beam, the volume holograms corresponding to the different incidence angles can be recorded separately into the medium, which is referred as angular multiplexing holograms. With this angular multiplexing technology of volume holographic storage, several images can be stored in the same volumetric site in the form of the volume holograms. Using angular multiplexing technology and optical configuration of Fourier transform hologram that has an advantage of smaller recording area, high density digital data can be implemented in photorefractive crystals.

\subsection{Mechanism of holographic storage in photorefractive crystals}

Holographic recording in photorefractive crystals is based on the effect of light-induced refractive index changes in photorefractive crystal, so called as photorefractive effects. During recording holograms, when both recording light beams interfere to generate bright and dark distribution in a photorefractive crystal, the light pattern of interference fringes can be transposed into refractive index pattern. The mechanism is based on that the charge carriers (usually electrons in $\mathrm{Fe}: \mathrm{LiNbO}_{3}$ ) in inorganic electro-optic crystals can be excited by a light of appropriate wavelength and become moveable therein ${ }^{[4,5]}$. The charge carriers are excited in the bright region of the interfering light pattern, and then migrate and subsequently trapped at other sites. Based on above-mentioned charge transport processes, the distribution of an electronic space-charge field is built up and is identical to the pattern of interference fringes. Accordingly, a modulation pattern of refractive index is formed via the electro-optic effect in the crystal, namely the photorefractive grating or hologram, which is consistent with the spatial distribution of interfering light field. Thus, the information of original object light is recorded into the photorefractive holographic grating.

\subsection{Angle-multiplexing holographic recording and retrieve}

In the holographic recording, it is required that an expanded and collimated laser beam is split into two parts via one beam splitter, in which one beam spatially modulated by a page of information after passing through a spatial light modulator (SLM) is called as object beam, and the other is a reference beam that defines the address where the information is to be stored. The reference beam provides an identity for the page carried by an object beam with different angles. If the reference beams with different angles are used for storage of the pages in the same volume of the 
holographic medium, the information on individual page can be distinguished from other pages by angle-addressing. This method of volume holographic storage with angle addressing mechanism is termed as angle-multiplexing holographic storage. In the optical configuration, the addressing mechanism for holographic recording is the incident angle of the reference beam, in which the reference angle is changed with each hologram recording. The reference beam is a plane wave, and the reference beams and the optical axis of object beam are all on the same plane.

A volume medium such as a Fe:Ce: $\mathrm{LiNbO}_{3}$ crystal is used for volume holographic recording, in which the recorded hologram has the effect of volume diffraction, namely Bragg diffraction. In retrieve each of pages of information from the volume holograms that has been recorded in the crystal, the reference beam that has record that page is employed to illuminate the recording crystal, with the same addressing angle as recording. According to the selectivity of Bragg diffraction, only one page that is recorded with this reference beam can be readout from the corresponding hologram, although the light interacts with all of the recorded holograms. Bragg's condition of volume grating diffraction can be expressed as

$$
2 n \Lambda \sin \theta=\lambda
$$

Where $\Lambda$ is grating constant, $n$ is refractive index of the medium, and $\lambda$ is wavelength of incident light in a vacuum. The angle $\theta$ between the incident beam and the entrance surface is called Bragg angle.

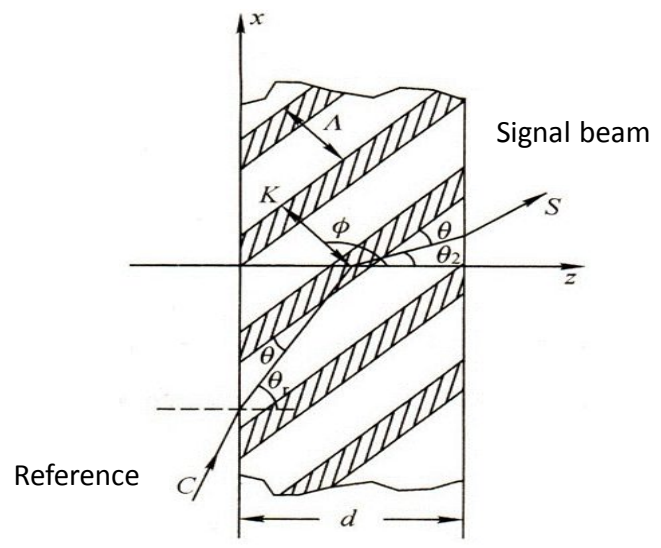

Fig.1 The diagram of Bragg diffraction of volume grating with a reference beam

The diagram of the diffraction of volume grating with a reference beam is shown as Fig. 1. According to three-dimension diffraction theory, when the reference beam is incident with Bragg's condition, the amplitude of diffraction wave from the matched holographic grating reaches the maximum, the matched page of information is retrieved completely. The illumination of the reference beam that does not match Bragg's condition will pass through the holographic grating (hologram) without diffraction. Thus, for retrieval of a particular page from the recorded multiplexing holograms, the angel addressing mechanism of reference beams can be used to access to the hologram. 


\section{OPTICAL CONFIGURATION AND EXPERIMENT}

The purpose of the experiment of angle-multiplexing holographic storage in teaching is to demonstrate the comprehensive application of optical knowledge and technology. The optical system and user control interface of the experiment is designed for teaching purpose.

The optical setup for storage of volume holograms is shown in Fig. 2. The recording medium is a cube-like Fe:Ce: $\mathrm{LiNbO}_{3}$ crystal of $8 \mathrm{~mm}$ side-size. The holographic gratings are recorded with a planar reference beam and signal beam at the wavelength of $633 \mathrm{~nm}$. The polarization states of an object beam and a reference beam should be parallel with each other to form interference when they overlap in the crystal. The half wave plate is used to adjust the intensity ratio of the object and reference beams, commonly about an equal ratio of them.

In the reference arm, a galvanometric scanner and a 4-F optical system with a pair of lenses are combined as angle-addressing component. The focal length of the lenses in 4-F optical system each is $75 \mathrm{~mm}$. When the reference beams pass through this angle-addressing component, all of the reference beams even with different incident angles can be illuminate on the same site of the crystal. The holographic recording geometry is $90^{\circ}$ geometry, in which the reference and object beams are incident on the two normal faces of the cube-like medium. In the signal arm for holographic recording, the Fourier-plane geometry is used for recording Fourier holograms with slightly off-focus, in which the SLM is Fourier-transformed onto the crystal. Furthermore, in the readout process, the signal wave reconstructed from holograms in turn is Fourier-transformed onto the CCD to capture the image. As shown in Fig. 2, a Fourier-transform lens L2 is placed behind the recording crystal to imaging the diffracted plane wave onto the CCD detector. In the experiment, the SLM, shutters and the CCD detector are controlled and operated via a user control interface with a LabView programming software.

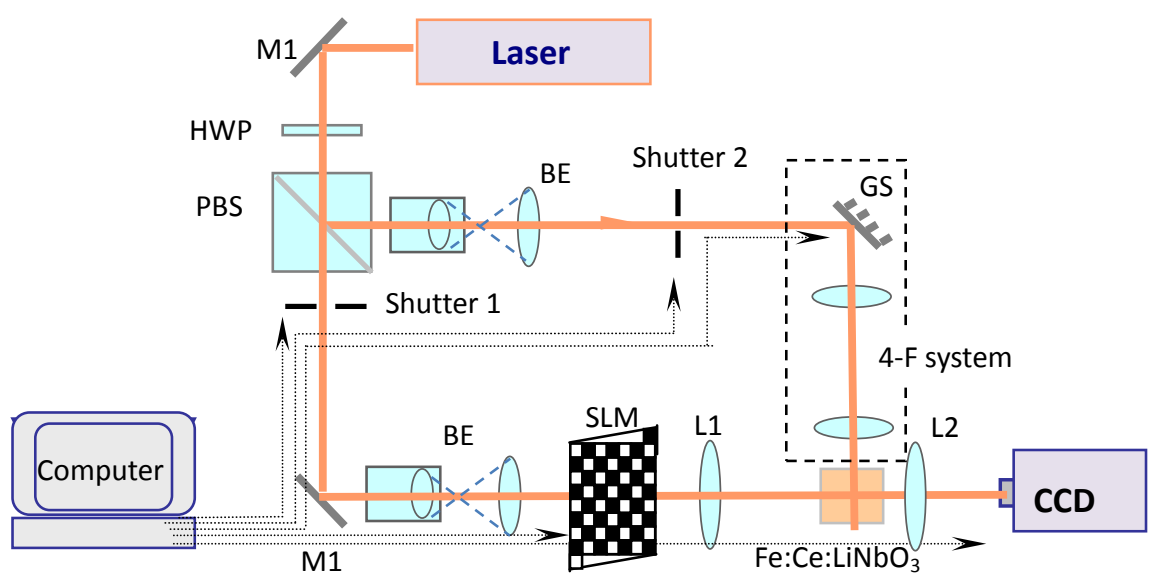

Fig.2 The schematic of angle-multiplexing holographic storage, PBS: polarizing beam splitter.

HWP: half wave plate. M: mirror. BE: beam-expander. GS: galvanometric scanner

In the experiment, the pages of information are generated by using a SLM that is an electronic binary object. The SLM made of such as liquid crystal display is used to display a two-dimensional binary pattern. A laser beam through the SLM becomes modulated in its two dimension pattern, which is commonly called as object wave beam. When the different 
patterns are loaded on the SLM respectively, object wave coming from the SLM carries the different page of information. Herein, the page means a two-dimensional spatial signal, or a two-dimensional pattern. The lens L1 is placed between the SLM and the recording crystal and is slightly off-focus from the crystal, so that the quasi-Fourier transform of the SLM pattern is recorded, shown as in Fig.3.

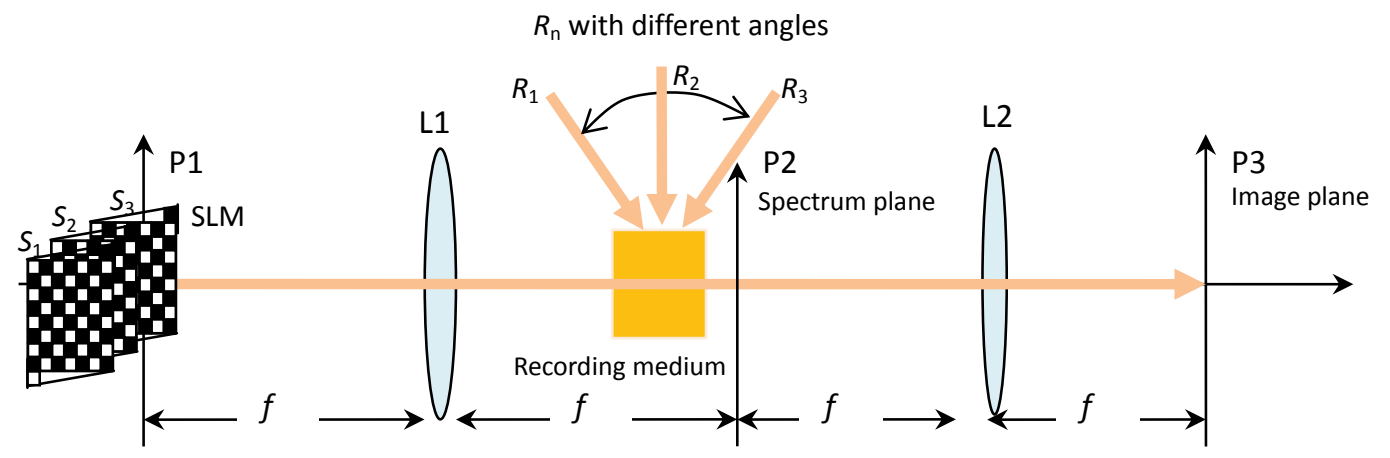

Fig.3 Angle-multiplexing optical configuration for quasi-Fourier transform of SLM pattern

In holographic recording, the exposure time for recording is dependent on the photorefractive response time of the crystal, also called as writing time. Students can try to enter the different values of exposure time in the user interface to implement holographic recording. After finished recording, the reconstruct image can be readout into the CCD detector and display on computer screen. If the original reference beam is employed to read the hologram, the page of information can be retrieved completely. Otherwise, even if the reference beam is deflected a little angle from its original recording angle, the diffraction efficiency and information of the page will be sharply declined. Fig. 4 (b) and Fig. 4 (c) show the retrieval images with the original recording angle and with a deflected angle. The image contrast of the latter is of dramatic decline.

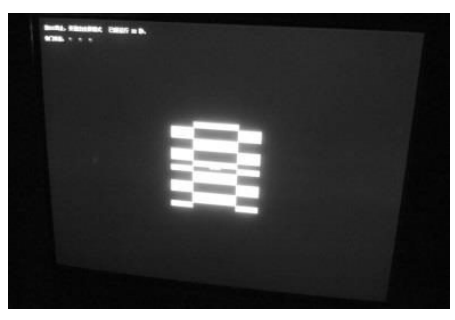

(a)

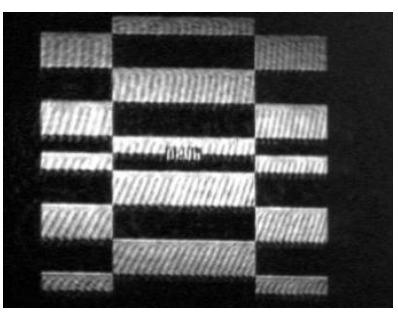

(b)

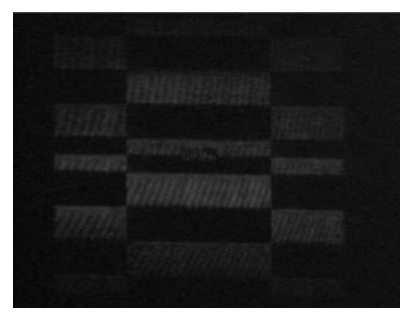

(c)

Fig.4 The effect of readout angle on the retrieval images: (a) pattern on SLM, (b) image readout with a reference recording angle, (c) image readout with a deflected angle 


\section{CONCLUSIONS}

In this paper, the experiment of volume holographic storage for teaching and training the practical ability of senior students in optics or optical engineering is introduced. The principle and experimental configuration of multiplexing holographic recording and readout based on Bragg selectivity of volume holographic grating is described in detail. With the different recording angles of reference beam, the holograms can be multiplexed to be recorded into photorefractive crystal, and the images of object can be read out from these holograms via angular addressing by using the original

reference beam. In this system, the experimental data acquisition and the control of the optoelectronic devices, such as the shutter on-off, image loaded in SLM and image acquisition of a CCD sensor, are automatically realized by using LabVIEW programming.

The students can learn to use advanced optoelectronic devices and the automatic control means via this experiment, and further understand the theoretical knowledge of optical information processing and photonics disciplines that have been studied in some courses. This experiment provides effective for students to understand concept and principle of optical information processing. In this experiment course, students are trained to master the professional operating skill and the data processing method in optoelectronic and optical information technology experiments. Students are expected to consolidate their theoretical knowledge in applied physics, and to learn using some advanced photoelectric detection devices such as SLM and CCD detector via understanding the operation mechanisms and applications of spatial light modulator and CCD detector. Furthermore, students can practice the measurement for diffraction power data and image acquisition via automatic control with a computer in the experiment of volume holographic storage.

\section{REFERENCES}

[1] G. W. Burr, F. H. Mok, D. Psaltis, "Angle and space multiplexed holographic storage using 90 geometry," Opt. Commun. 117, 49-55 (1995)

[2] J. H. Hong, I. C. McMichael, T. Y. Chang, W. R. Christian, E. G. Paek, "Volume holographic memory systems: techniques and architectures," Opt. Eng. 34, 2193 2203 (1995)

[3] G. W. Burr, C. M. Jefferson, H. Coufal, M. Jurich, J. A. Hoffinagle, R. M. Macfarlane, R. M. Shelby, "Volume holographic data storage at an areal density of 250 gigapixels/in. ${ }^{2}$," Opt. Lett. 26, 444-446 (2001)

[4] H. J. Coufal, D Psaltis, G. T. Sincerbox, [Holographic data storage], Springer, 21-31 (2000)

[5] S. Tao, Z. Jiang, Y. Wan, D. Wang and Y. Wang. [Volume holography and applications], Science Press, Beijing 61-68 (2013)

[6] J. W. Goodman, [Introduction to Fourier Optics], Roberts \& Company Publishers, (2004)

[7] N. Lu, [Fourier optics], China Machine Press, Beijing, (2006) 